${ }^{1}$ Centro de Investigación e Innovación en Odontología Clínica (CIDIC), Facultad de Odontología, Universidad de La Frontera. Temuco, Chile. ${ }^{2}$ Departamento de Periodoncia, Facultad de Odontología, Universidad de los Andes. Santiago, Chile. Unidad de Reumatología, Facultad de Medicina, Universidad de los Andes. Santiago, Chile. ${ }^{4}$ Unidad de Reumatología, Facultad de Medicina, Universidad de La Frontera. Temuco, Chile. ${ }^{a}$ Cirujano Dentista. boctor en Ciencias Odontológicas (PhD). 'Magister en Ciencias Odontológicas mención Periodontología (MSc).

Trabajo no recibió financiamiento. Los autores declaran no tener conflictos de interés.

Recibido el 31 de agosto de 2018, aceptado el 1 de febrero de 2019.

Correspondencia a: Víctor Beltrán Varas Fono: $45-2235775$ victor.beltran@ufrontera.cl

\section{¿La periodontitis crónica modifica la morbilidad de la artritis reumatoide?: Aspectos clínicos y moleculares. Una revisión sistemática}

\author{
SCHILIN WEN ${ }^{1, a}$, VÍCTOR BELTRÁN ${ }^{1, a, b}$, ALEJANDRA CHAPARRO $^{2, a, c}$, \\ FRANCISCO ESPINOZA ${ }^{3}$, JUAN PABLO RIEDEMANN ${ }^{4}$
}

\section{Association between chronic periodontitis and rheumatoid arthritis.} A systematic review

\begin{abstract}
Rheumatoid arthritis $(R A)$ and chronic periodontitis $(C P)$ may be related due to a bidirectional etiology. The evidence shows that CP could alter the clinical course of $R A$. We performed a systematic search to determine if $C P$ alters the morbidity of RA, analyzing its clinical and molecular aspects. Of 552 initial articles found, 16 were selected for a thorough review. There is a greater prevalence of $C P$ in patients with $R A$. Patients with $R A$ have significantly higher values of periodontal clinical parameters than healthy controls. Arthritis activity is significantly greater in patients who suffer from $C P$ and decreases with nonsurgical periodontal treatment. There is a significant relationship between the severity of $C P$ and $R A$ activity.
\end{abstract}

(Rev Med Chile 2019; 147: 762-778)

Key words: Arthritis, Rheumatoid; Chronic Periodontitis; Comorbidity.
L a artritis reumatoide (AR) es una enfermedad crónica, autoinmune e inflamatoria que afecta principalmente a las articulaciones sinoviales ${ }^{1}$. Presenta una incidencia entre $0,5 \%$ y $1 \%{ }^{2}$ a nivel global y a nivel nacional su prevalencia en población mayor a 15 años se aproxima a $0,46 \%{ }^{3}$.

La AR se caracteriza por una disminución de la actividad física, deterioro en la calidad de vida y riesgo acumulativo de comorbilidades, lo que la convierte en una afección de gran impacto para el individuo y la sociedad ${ }^{4}$. En virtud de lo anterior, es primordial establecer los factores que influyen en su etiología y los factores de riesgo implicados en su desarrollo. La edad avanzada, tabaquismo regular, diabetes mellitus, obesidad y osteoporosis se han asociado positivamente con $\mathrm{AR}^{5}$.

Para determinar la actividad de la AR se emplean diversos índices. El puntaje de actividad de la enfermedad para artritis reumatoide o DAS 28 (por las siglas del inglés Disease Activity Score) es uno de los más utilizados e incluye el número de articulaciones inflamadas o sensibles de entre 28 articulaciones posibles, la evaluación global por el paciente y marcadores de inflamación que pueden ser la proteína $C$ reactiva (PCR) o la velocidad de sedimentación de eritrocitos (VHS) ${ }^{6}$. De acuerdo a sus resultados, que pueden ir de 0 a 9,4 , permite la clasificación de la AR en: remisión de la enfermedad $(<2,6)$, actividad baja $(\geq 2,6 \mathrm{y}<3,2)$, moderada $(3,2$ a 5,1$)$ o alta $(>5,1)^{7}$.

Otros índices de actividad de enfermedad son el Clinical Disease Activity Index (CDAI) y el Simplified Disease Activity Index (SDAI). Ambos también incluyen el número de articulaciones inflamadas y sensibles (de entre 28 posibles), una evaluación global por el paciente y una evaluación global del médico (CDAI). Si además se agrega 
un marcador de inflamación como la PCR, se denomina índice de actividad simplificado de la enfermedad (SDAI) ${ }^{8}$. Sus resultados permiten clasificar el estado de la enfermedad como en actividad alta ( $>22)$, moderada (10 a 22), baja $(<10)$ y en remisión $(<2,8)^{9}$.

Por otra parte, la periodontitis crónica (PC) es también una afección inflamatoria crónica ampliamente extendida en la población, caracterizada por la destrucción del tejido de soporte óseo de los dientes y que en sus formas graves puede causar la pérdida de estos ${ }^{10}$. El $75 \%$ del gasto por concepto de enfermedades dentales se destina al tratamiento de la periodontitis, afectando a $11 \%$ de la población adulta mundial ${ }^{11}$. A nivel nacional, se ha observado que el número de sitios con pérdida de inserción mayor a $6 \mathrm{~mm}$ es $38,7 \%$ en población adulta joven y $69,4 \%$ de los sitios en el adulto mayor ${ }^{12}$.

Su etiología es causada por patógenos periodontales, principalmente bacterias de tipo anaerobias Gram (-), que logran colonizar y organizarse en un biofilm subgingival causando disbiosis en el hospedero susceptible. Entre las bacterias patógenicas que conforman este biofilm, Porphyromonas gingivalis ( $P$. gingivalis) sintetiza la enzima peptidil arginina deiminasa $(\mathrm{PAD})$, que en condiciones inflamatorias patológicas se ha relacionado con la disminución o perdida de la tolerancia inmune a proteínas citrulinadas, favoreciendo el desarrollo de AR en individuos susceptibles ${ }^{13}$. Además, se ha sugerido que la PC estaría involucrada en la producción de anticuerpos antiproteínas citrulinadas (ACPA $)^{14}$, un marcador específico de la AR que se puede detectar incluso años antes del inicio de la enfermedad y su presencia se correlaciona fuertemente con gravedad de la $\mathrm{AR}^{15,16}$.

El objetivo de la presente revisión fue evaluar si la PC puede modificar la morbilidad de la AR, a través del análisis de los aspectos clínicos y moleculares implicados en la etiopatogenia de ambas patologías.

\section{Material y Método}

Se utilizó la base de datos MEDLINE y en una primera etapa se realizó una búsqueda sensible utilizando términos $\mathrm{MeSH}$ y términos libres con las palabras claves "rheumatoid arthritis", "chronic periodontitis" y "periodontitis", en la que se utilizaron operadores booleanos (AND y OR), obteniéndose 552 artículos en total.

A continuación, 2 revisores de manera independiente evaluaron cada uno de los títulos y resúmenes, eliminando los duplicados y aplicando los criterios de selección: 1) Estudios observacionales y clínicos de los últimos 10 años: desde enero de 2008 a junio de 2018; 2) en humanos; 3 ) estudios que evaluaran la relación entre PC y la morbilidad de AR y 4) en idioma inglés. De esta selección se obtuvieron 43 artículos. Luego se realizó el análisis de texto completo, descartándose 27 artículos por no tener relación con el objetivo específico de estudio. Finalmente, 16 artículos fueron seleccionados por atingencia al objetivo del estudio (Figura 1).

\section{Resultados}

\section{Aspectos clínicos de la asociación}

\subsection{Datos epidemiológicos (Tabla 1)}

Uno de los estudios analizados determinó que la ocurrencia y la gravedad de la PC son mayores en sujetos con $\mathrm{AR}$, observándose que la $\mathrm{PC}$ moderada fue más prevalente en pacientes con AR (58\%), en comparación con el grupo control $(7,2 \%)^{17}$. Similares fueron los resultados obtenidos por Menke de Smit et al. ${ }^{18}$ que evaluaron la condición periodontal en 95 pacientes con AR, utilizando el índice de detección periodontal holandés para necesidades de tratamiento (DPSI), el cual evalúa sangrado al sondaje, nivel de inserción clínica y profundidad al sondaje. Los resultados indican que $43 \%$ de los pacientes con AR presentaba PC moderada y $27 \%$ presentaba PC grave, siendo más prevalentes en AR en comparación con controles sanos $(p<0,001)$.

Mikuls et al. ${ }^{19}$ observaron que $35 \%$ de los pacientes con AR y 26,4\% de controles sanos presentaron PC. Por tanto, la prevalencia de PC en pacientes con AR fue también mayor en comparación con los controles ( $\mathrm{p}=0,022)$.

Similares fueron los resultados de Lee et al. ${ }^{20}$ y Choi et al. ${ }^{21}$, en los cuales la PC moderada fue observada en $61,7 \%$ y $61 \%$ de los pacientes con $A R$, respectivamente $(p<0,001)$. Estos resultados han sido confirmados recientemente en el estudio de Mobini et al. ${ }^{22}$, donde $63,5 \%$ de los pacientes con AR padecía PC y 12,2\% PC moderada-grave, siendo la $\mathrm{PC}$ más prevalente en AR en compara- 


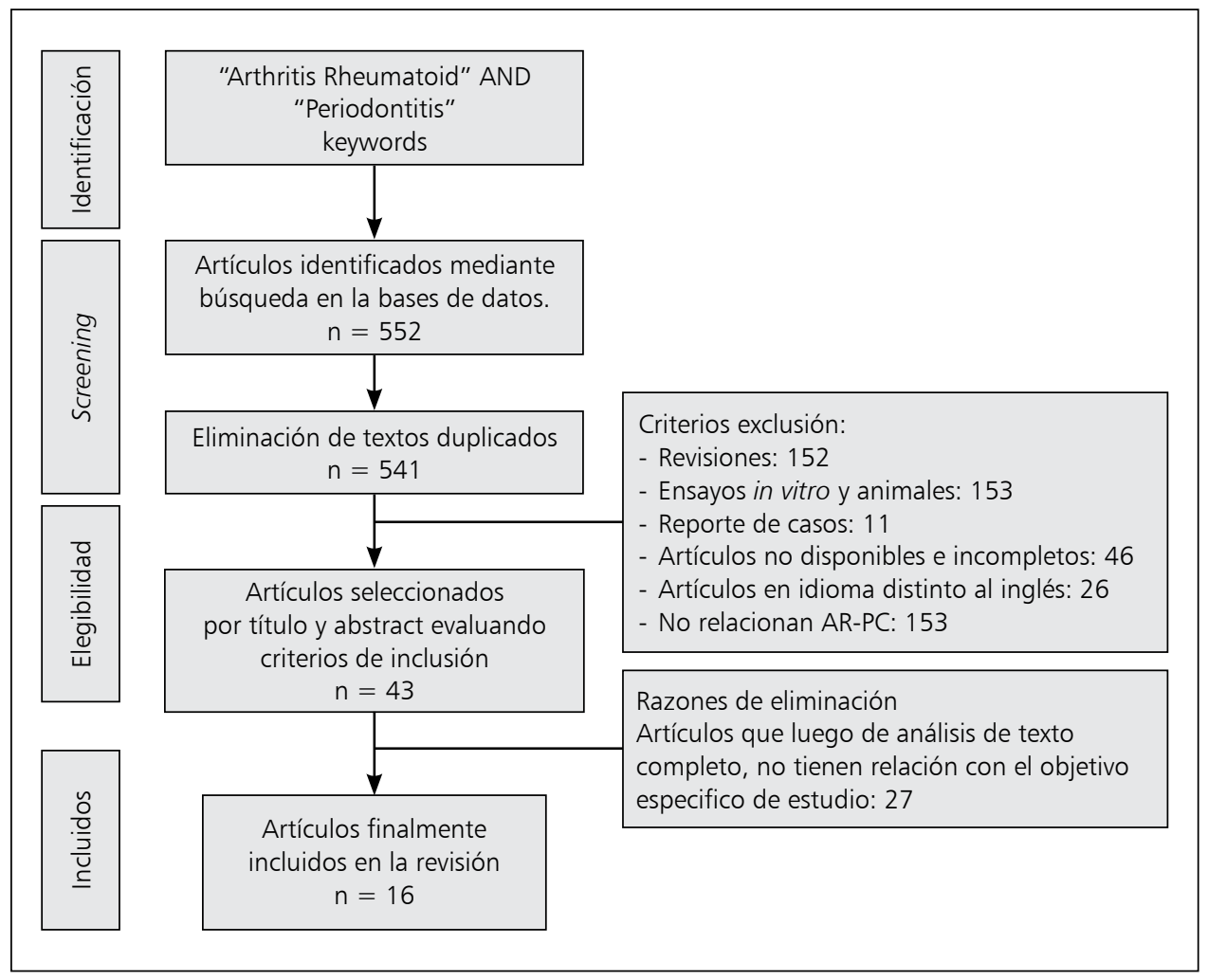

Figura 1.

ción con controles. Por otra parte, Chou et al. ${ }^{23}$ investigaron el riesgo de AR asociado a la exposición a PC en una cohorte compuesta por un millón de participantes seleccionados al azar durante el año 2010 en Taiwán. El riesgo de AR reportado en pacientes con PC fue 1,33 (IC del 95\% 1,08-1,64).

\subsection{Relación entre PC y actividad de AR (Tabla 2)}

Mikuls et al. ${ }^{19}$ evaluaron la actividad de artritis utilizando el índice DAS28 en 287 pacientes con AR. Los resultados muestran que aquellos pacientes con PC obtuvieron mayores puntajes en índice DAS28, mayor recuento de articulaciones sensibles y mayor daño radiográfico en comparación con los pacientes sin PC.

En otro estudio realizado en 95 pacientes con $\mathrm{AR}$, se observó que aquellos sujetos con PC grave presentaron mayores puntajes en comparación con los pacientes con PC moderada o sin PC $(\mathrm{p}<0,001)^{18}$.

Hashimoto et al. ${ }^{24}$ evaluaron si PC y P. gingivalis se asociaban a la actividad de AR durante la fase preclínica de la enfermedad. En 72 pacientes con altralgia, que no se encontraban en tratamiento para la AR, se observó que aquellos sujetos con PC presentaron mayor actividad de AR y, por lo tanto, un riesgo aumentado de indicación del medicamento metotrexato, incluso después del ajuste de factores de variables confundentes.

\subsection{Relación entre parámetros clínicos periodontales y AR (Tabla 3)}

Los parámetros clínicos analizados fueron índice de placa (PI), índice gingival (GI), profundidad de sondaje (PPD), sangrado al sondaje (BOP), nivel de inserción clínica (CAL) e índice de higiene oral simplificado (IHO-S). Existen hallazgos interesantes en cuanto a los parámetros clínicos periodontales. Se han observado mayores valores en pacientes con AR en comparación con controles sanos ${ }^{17,20}$. Pacientes con AR presentan mayor porcentaje de sitios con PPD mayor o igual $5 \mathrm{~mm}$ en relación a controles ${ }^{19}$. Además, estos índices de inflamación y destrucción periodontal se correlacionan positivamente con la actividad de AR medida por DAS28 $(\mathrm{p}<0,01)^{25}$. Sin embargo, existen estudios recientes en los que no se ha observado relación ${ }^{22,26}$. 


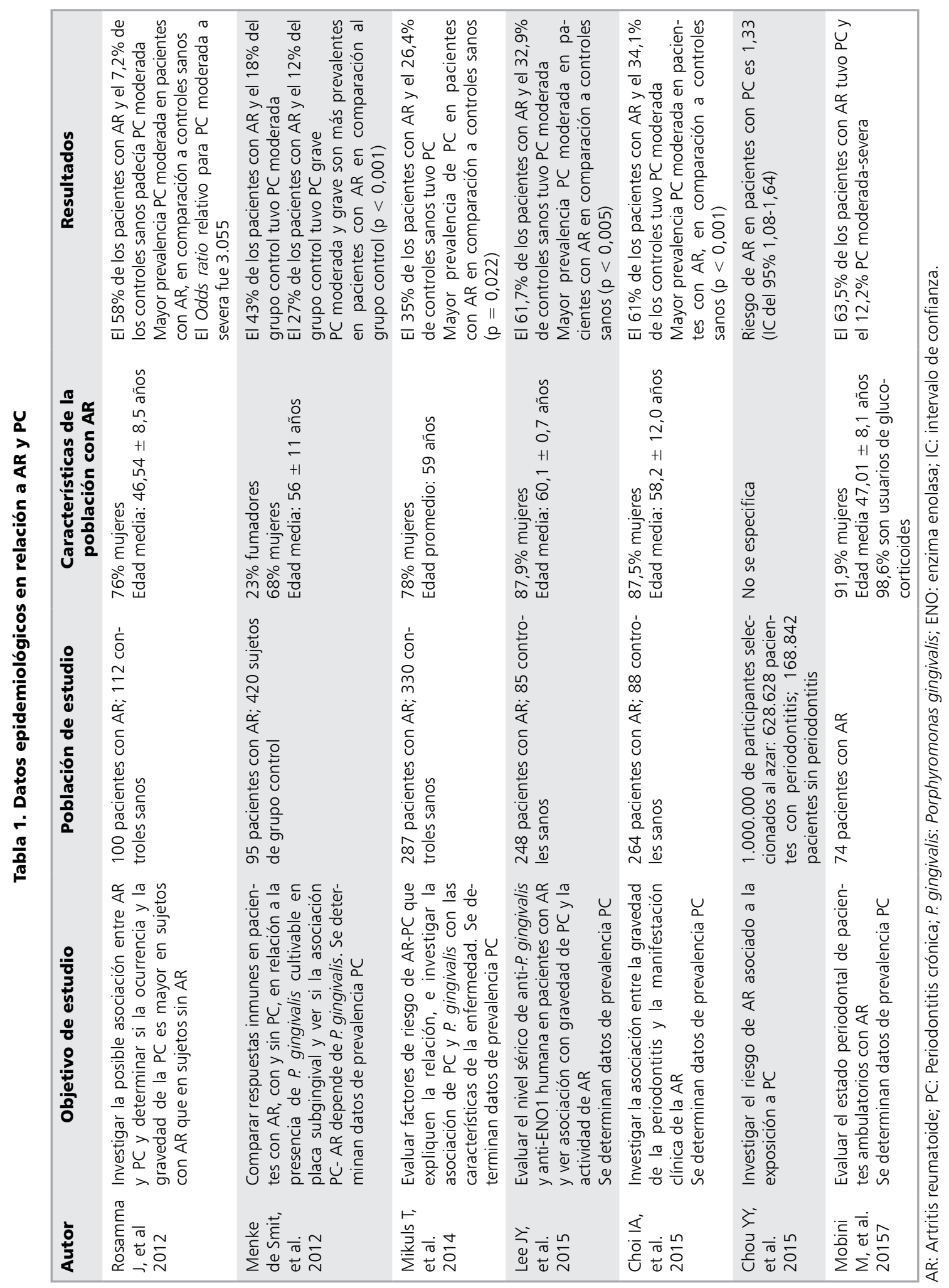




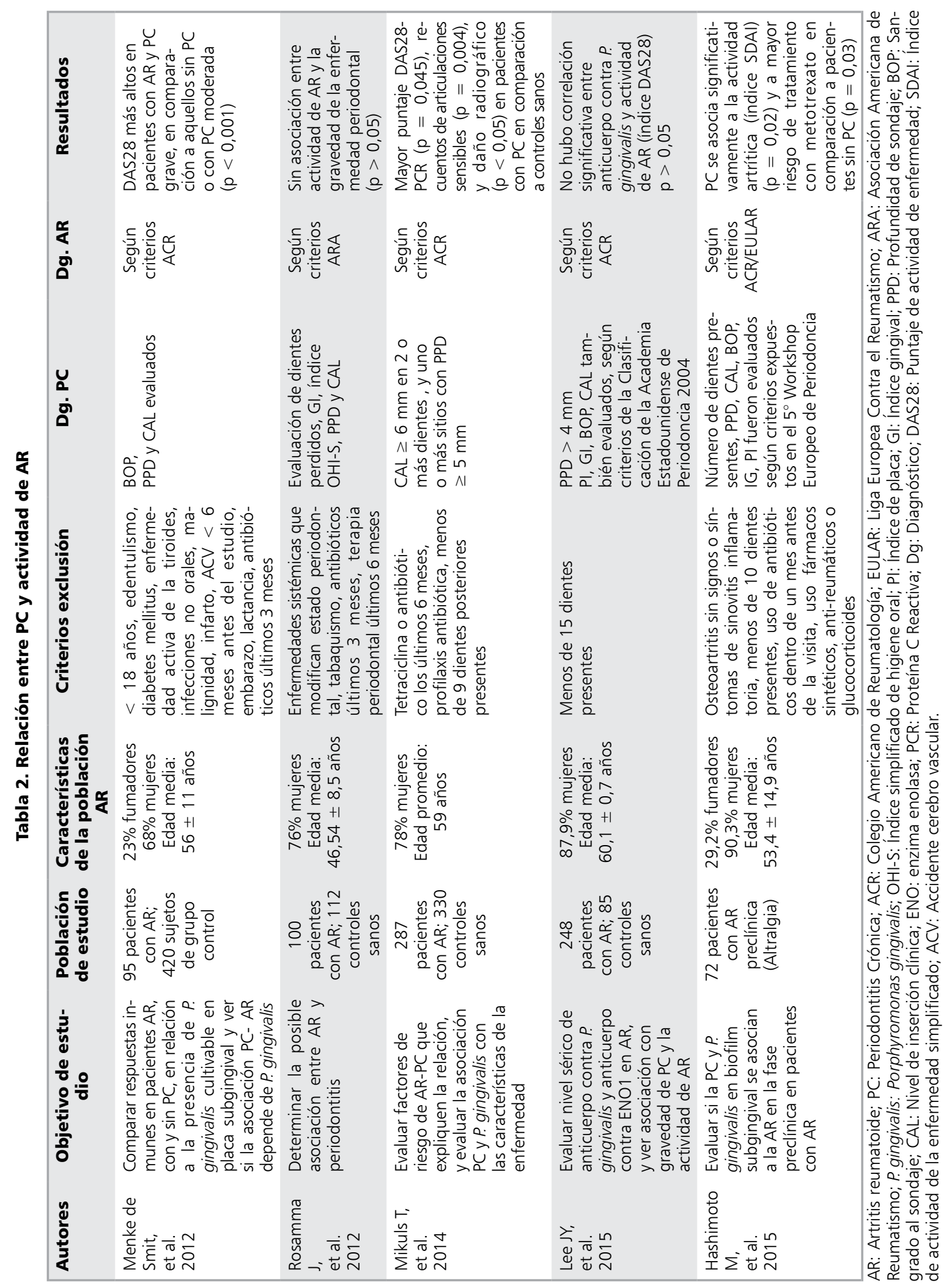




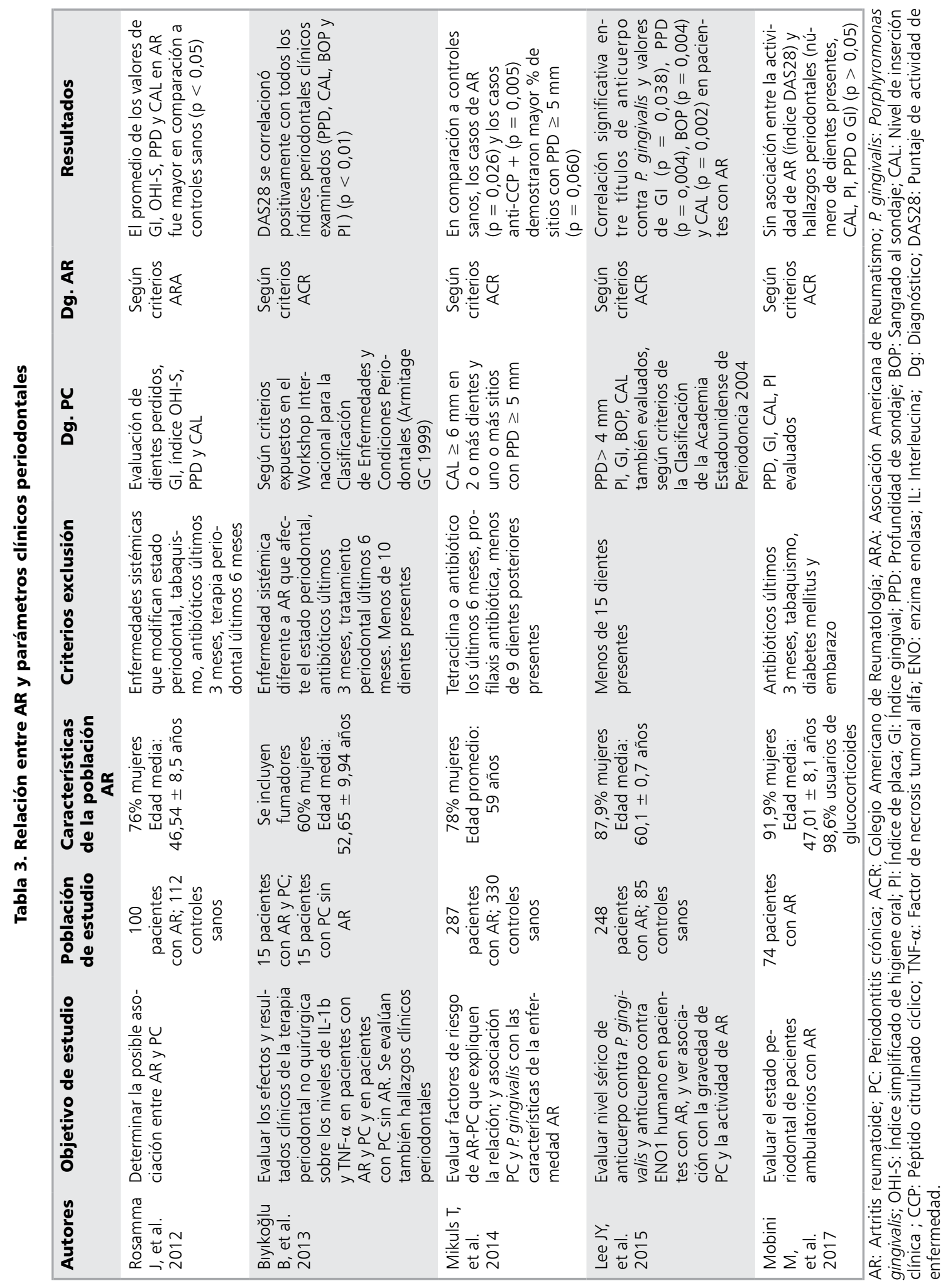


Choi et al. ${ }^{21}$ observaron que BOP y GI se correlacionaron con la duración de la $\mathrm{AR}(\mathrm{p}<0,001)$, no así PPD y CAL, que no mostraron diferencias significativas. Es interesante mencionar que en el mismo estudio, los pacientes con AR crónica (que tenían una duración de AR mayor o igual a 10 años) mostraron valores de GI y BOP significativamente más altos $(\mathrm{p}<0,001)$ que los controles, a diferencia de los pacientes con AR temprana (con una duración de AR menor a 1 año) que no mostraron resultados significativos. Esto reafirma el elevado nivel de inflamación presente en etapas avanzadas de la enfermedad.

\subsection{Efectos del tratamiento periodontal no quirúrgico en AR (Tabla 4)}

Se ha observado que la actividad de AR medida por DAS28 disminuye luego de la terapia periodontal no quirúrgica ${ }^{25,27}$. En este contexto, Erciyas et al..$^{28}$ estudiaron los efectos del tratamiento periodontal en los parámetros periodontales clínicos y en los reactantes de fase aguda de pacientes con AR de actividad baja y moderada-alta de la enfermedad. En ambos grupos se observó una disminución de PCR y VHS a los 3 meses de tratamiento.

Shimada et al. ${ }^{29}$ también observaron en sus resultados que existe una mejoría de la condición periodontal y la actividad de la enfermedad AR $(\mathrm{p}<0,05)$ al realizar instrucción de higiene oral y destartraje supragingival, sin embargo, los niveles séricos de inmunoglobulina $\mathrm{G}$ antipéptido citrulinado cíclico (IgG anti-CCP), IgG anti-PPAD y PAD-4 se mantuvieron sin variaciones. Por otra parte, en los resultados de Pinho et al..$^{30}$, el tratamiento periodontal no quirúrgico no logró diferencias significativas entre pacientes con AR y controles sanos. Los niveles de VHS y PCR se mantuvieron altos en pacientes con $A R$ pese al tratamiento periodontal.

\section{Aspectos moleculares: enzimas, anticuerpos y marcadores de AR (Tabla 5)}

\subsection{Relación entre niveles de anticuerpos \\ $P$. gingivalis $y A R$}

En PC, existe un aumento en la respuesta de anticuerpos contra $P$. gingivalis en pacientes con AR en comparación a controles ${ }^{18,20}$, sin embargo, no se ha demostrado una correlación entre los niveles de estos anticuerpos y las características clínicas de la enfermedad. Por el contrario, en los resultados de Seror et al. ${ }^{31}$ no se detectó relación entre los títulos de anticuerpos contra $P$. gingivalis y la AR o con ACPA positivo, sin embargo, se observó que aquellos pacientes con altos títulos de anticuerpos contra $P$. gingivalis fueron más propensos a desarrollar erosiones típicas relacionadas a la $\operatorname{AR}(\mathrm{p}=0,02)$.

Además, se ha demostrado que los anticuerpos contra PPAD se elevan en AR en comparación con los controles sanos, e incluso se elevan más que en aquellos pacientes con $\mathrm{PC}^{32}$.

\section{2. $P C$ y marcadores de enfermedad $A R$}

El BOP se correlaciona con elevados niveles de anticuerpo anti-CCP, aunque no se ha reportado relación con el factor reumatoide (RF) ni con $\mathrm{PCR}^{21}$. Sin embargo, se ha determinado que pacientes con PC presentan concentraciones más altas de RF circulante y de anticuerpo anti-CCP que aquellos sin PC. Sumado a esto, existe una correlación significativa entre el anticuerpo contra $P$. gingivalis, los anticuerpos anti-CCP $(\mathrm{p}=0,022)$, RF $(\mathrm{p}=0,001), y$ PCR $(\mathrm{p}=0,011)^{19}$.

Elevados niveles de anticuerpos antienolasa (anti-ENO) se han correlacionado con parámetros periodontales y una mayor proporción de casos de AR y ACPA positivo se han observado en $\mathrm{PC}^{20}$.

Por otra parte, se ha observado también que los niveles de anticuerpos contra $P$. gingivalis en pacientes con AR se relacionan positivamente con los títulos de anticuerpo anti-CCP, con anti-ENO y con $\mathrm{VHS}^{20}$.

\section{Discusión}

La evidencia científica actual establece una asociación entre AR y PC. Específicamente se reporta una mayor prevalencia de $\mathrm{PC}$ en pacientes con diagnóstico de $\mathrm{AR}^{19,22}$ y un mayor riesgo para el desarrollo de AR en pacientes con PC en comparación con la población general ${ }^{23}$. En este contexto, Mikuls et al. ${ }^{19}$ reportaron 35\% de prevalencia de $\mathrm{PC}$ en pacientes con AR, mientras que Mobini et al. ${ }^{22}$ describen $63,5 \%$.

Adicionalmente a estos antecedentes, otros investigadores describen que la prevalencia de PC moderada-grave es mayor en pacientes con AR al compararlos con controles sanos. Rosama et al. ${ }^{17}$ sugieren $58 \%$ de prevalencia de PC moderada-grave en pacientes con AR, Menke de Smit 


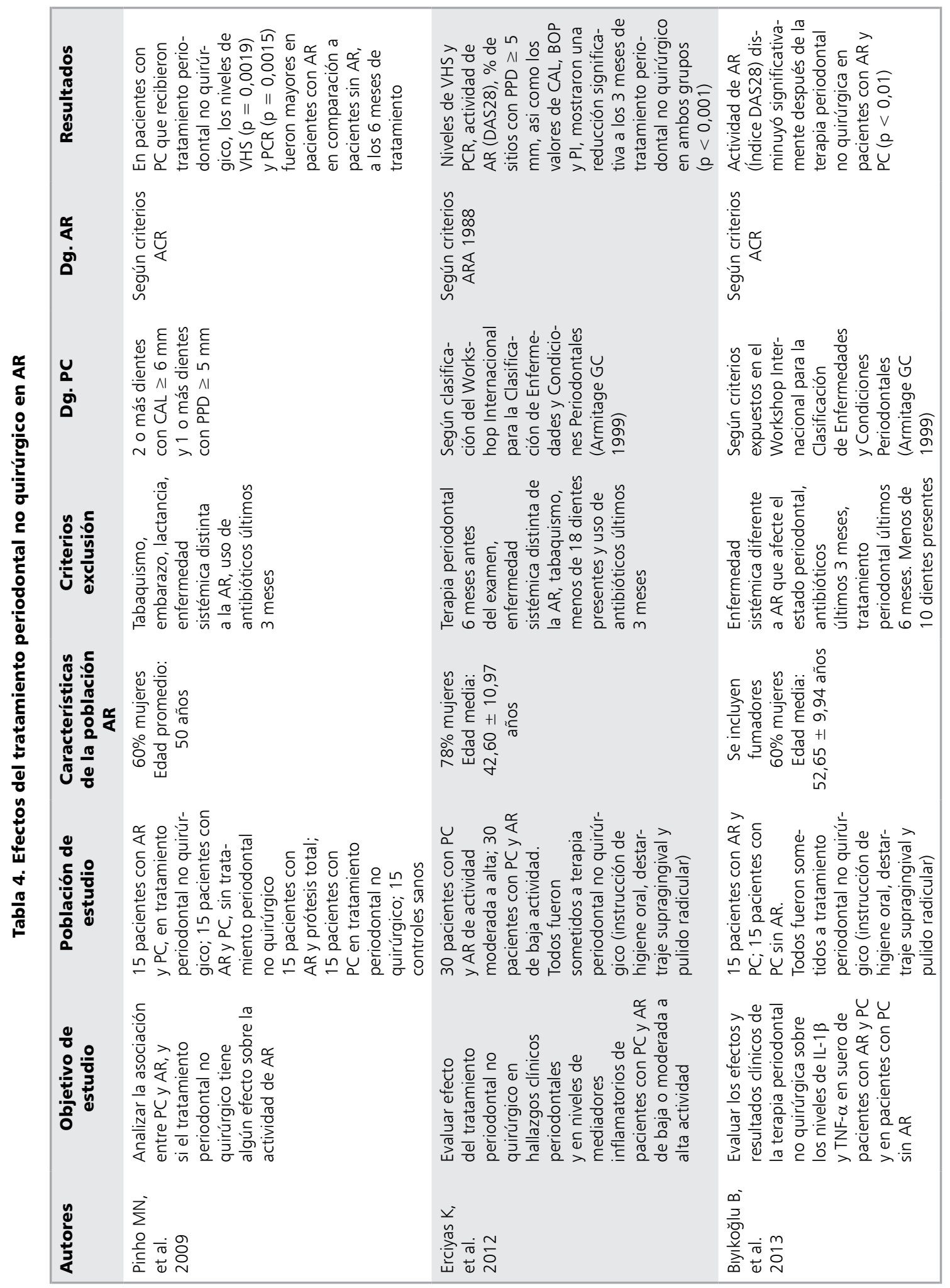




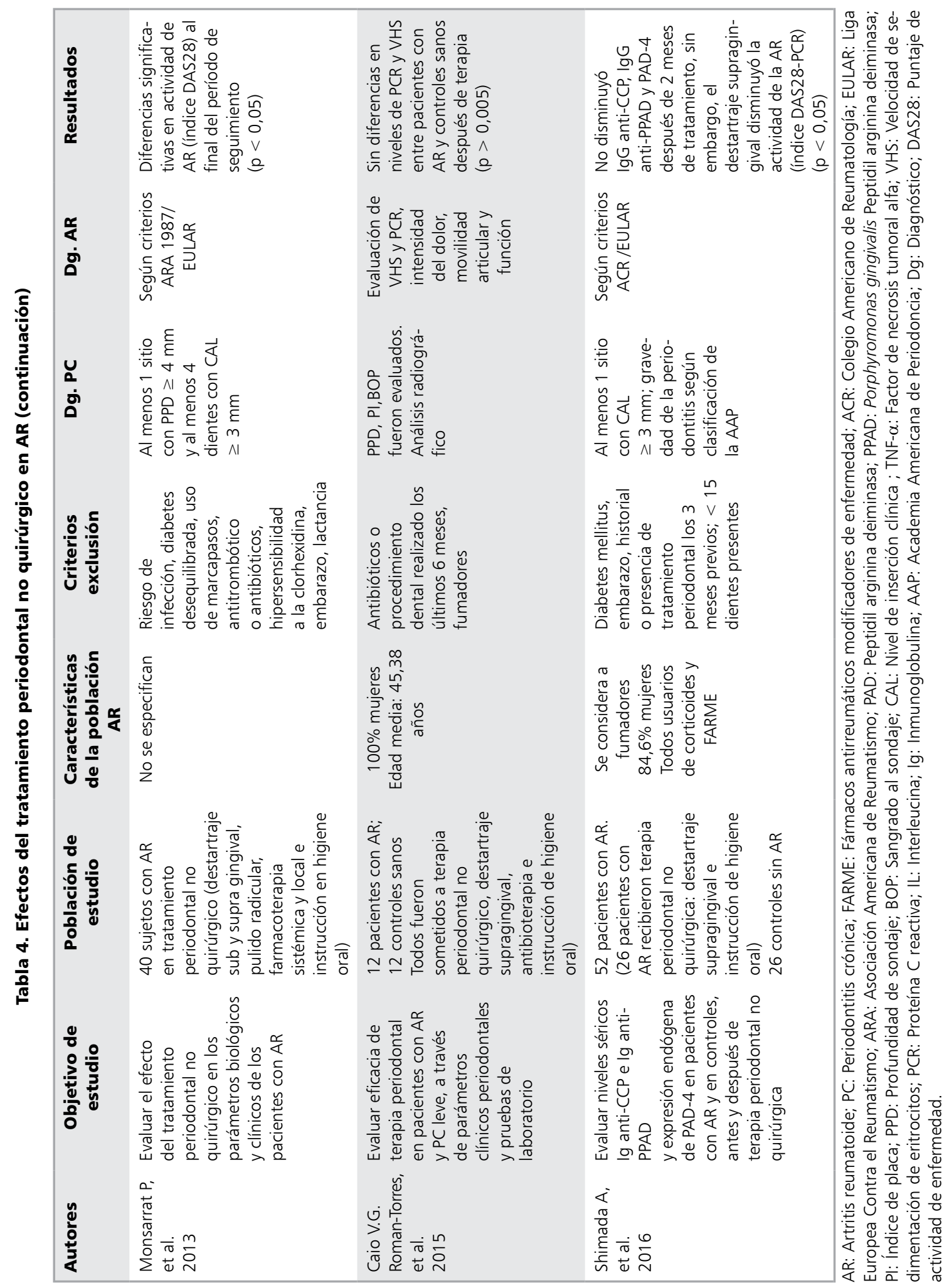




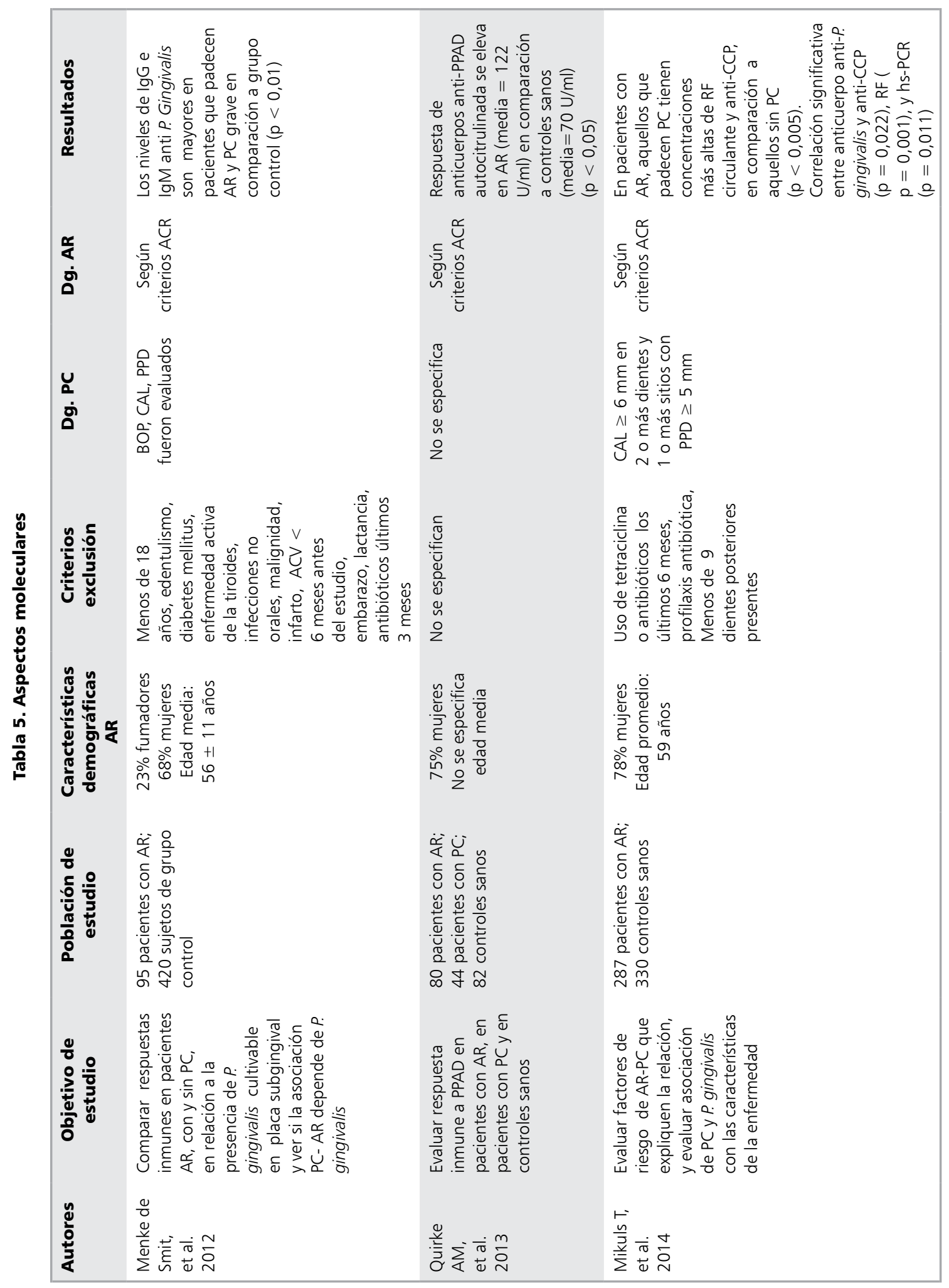




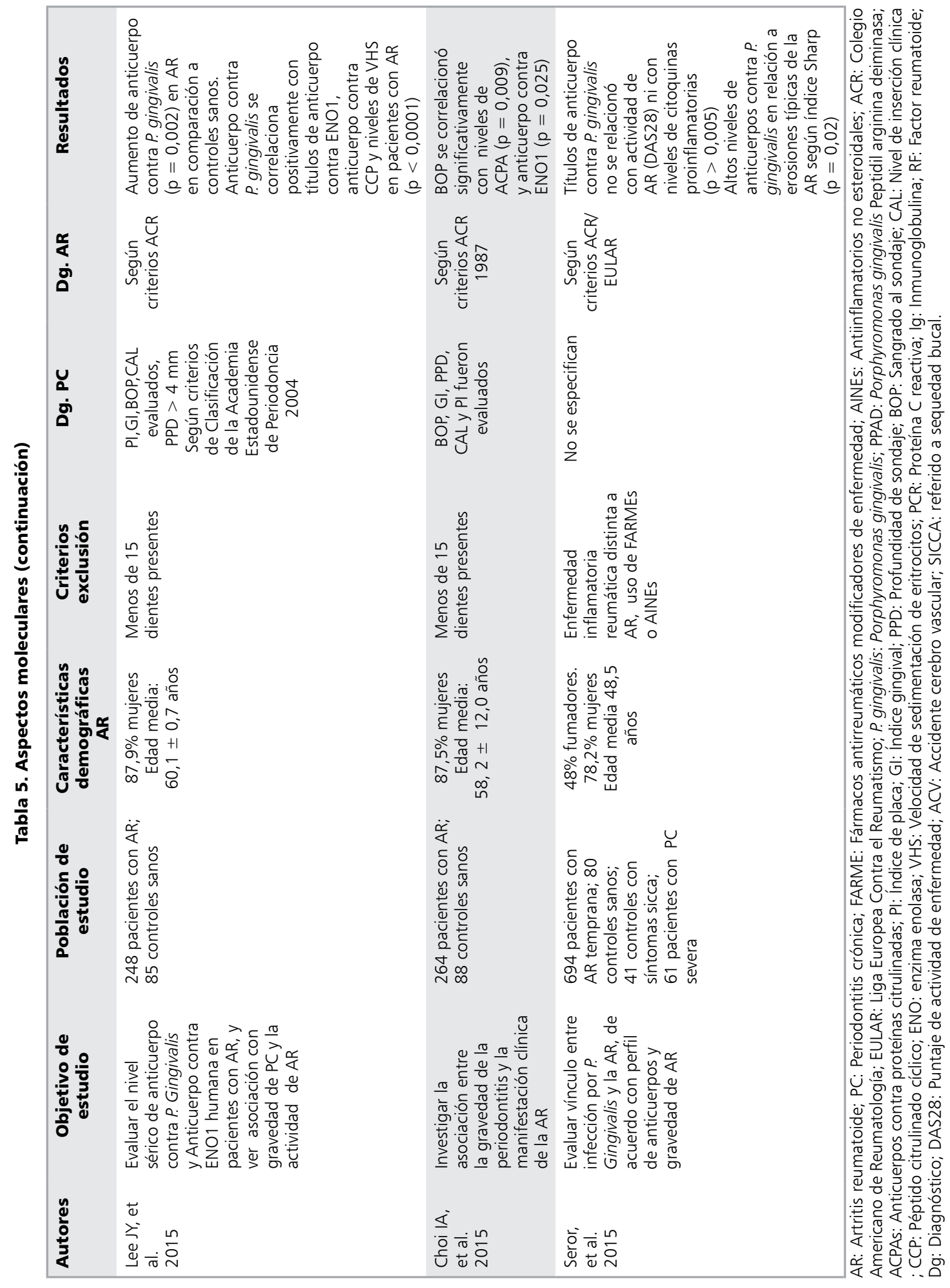


et al. ${ }^{18}, 43 \%$ de PC moderada y $27 \%$ de PC grave, mientras que Lee et al. ${ }^{20}$ y Choi et al. ${ }^{21}$ establecen $61,7 \%$ y $61 \%$ de PC moderada, respectivamente.

En relación a la actividad de AR, observamos que aquellos pacientes con PC presentan mayores puntajes de actividad de artritis en comparación con los pacientes sin $\mathrm{PC}^{19}$, y se ha observado una relación proporcional entre la severidad de PC y actividad de $\mathrm{AR}^{18}$. Inclusive, aquellos pacientes que presentan altralgia, síntoma clínico inicial de la $\mathrm{AR}$, y que no han sido tratados con medicamentos antirreumáticos, se describe que la actividad de AR es mayor en aquellos pacientes diagnosticados con AR y PC al compararlos con pacientes con AR solamente ${ }^{24}$. Estos resultados sugieren que la asociación entre AR y PC no es casual, y que sus mecanismos etiopatogénicos son similares en ambas patologías.

En relación a los hallazgos periodontales descritos en pacientes con AR, se destacan aumentos en los valores de PI, GI, PPD, BOP y CAL al compararlos con sujetos sanos, indicando una mayor destrucción periodontal en pacientes diagnosticados con $\mathrm{AR}^{17}$. Sin embargo, no se ha reportado asociación entre los parámetros clínicos periodontales y la actividad de la $\mathrm{AR}^{20}$.

Es interesante discutir acerca de los resultados del estudio de Rosama et al. ${ }^{17}$, en el cual todos los participantes recibieron prescripción de uso de antiinflamatorios no esteroidales (AINE), y, a pesar de ello, se establecieron mayores parámetros periodontales inflamatorios e índice de sangrado al sondaje periodontal en pacientes con AR al compararlos con los controles. De forma similar, Choi et al. ${ }^{21}$ sugieren una relación entre la gravedad de la inflamación periodontal y la duración de la AR, describiendo que pacientes con AR crónica presentan valores significativamente más altos de GI y BOP $(\mathrm{p}<0,001)$ que los controles, a diferencia de los pacientes con AR temprana, quienes no presentan diferencias significativas, confirmando el elevado componente inflamatorio en etapas avanzadas de la AR. Además, los parámetros periodontales inflamatorios se asociaron positivamente con los niveles de VHS y ACPAs, apoyando la teoría que los mecanismos inmune inflamatorios similares de desregulación inflamatoria, predisponen a que pacientes con diagnóstico de AR y PC desarrollen formas avanzadas, agresivas y graves de ambas patologías ${ }^{17}$.
Como antecedente adicional y que refuerza la plausibilidad biológica de la asociación, investigaciones reportan un aumento en los niveles de los anticuerpos contra PPAD a nivel sérico de pacientes con AR en comparación con controles sanos ${ }^{32}$, sugiriendo un alto nivel de proteínas citrulinadas en pacientes con AR y, dentro de este contexto, es relevante destacar que existe una mejoría de la actividad de la AR $(\mathrm{p}<0,05)$ en respuesta a la terapia periodontal convencional. Sin embargo, a nivel sérico no se observan variaciones de IgG anti-PPAD y PAD- ${ }^{29}$. Estos resultados sugieren que la respuesta frente a la terapia periodontal no invasiva produce una disminución local de los mediadores de inflamación, pero la citrulinación de proteínas involucra mecanismos más complejos, entre ellos, se ha planteado una pérdida de tolerancia a las proteínas citrulinadas en la AR, en la cual, los anticuerpos anti-PPAD podrían presentar un rol protector para el desarrollo de $\mathrm{PC}$ en pacientes con $\mathrm{AR}^{16}$.

Para finalizar, al analizar los principales resultados de otras revisiones sistemáticas que investigaron el efecto de la terapia periodontal convencional no quirúrgica sobre los parámetros clínicos moleculares de la AR, Kaur et al. ${ }^{33}$ concluyen que existe un buen nivel de evidencia científica para plantear la asociación entre AR y PC, especialmente en relación a la pérdida de piezas dentarias, pérdida de inserción clínica y tasa de sedimentación de eritrocitos y evidencia moderada en relación a la disminución de marcadores moleculares post terapia periodontal. El mismo autor, en una revisión posterior, Kaur et al. $^{34}$, sugieren disminuciones significativas de los títulos de TNF, VHS e índice DAS 28 en respuesta al tratamiento periodontal. De forma similar, Silvestre et al. ${ }^{35}$ concluyen que la terapia periodontal logra una disminución significativa en el índice DAS 28 y que parámetros bioquímicos moleculares como proteína $\mathrm{C}$ reactiva, IL-6 y TNF- $\alpha$ tienden a disminuir después de inicio de la terapia, pero de forma no significativa.

\section{Conclusión}

La evidencia científica demuestra que existe una mayor prevalencia de $\mathrm{PC}$ en pacientes con AR; los pacientes con AR presentan un aumento en la gravedad de parámetros clínicos periodontales; la 
actividad de AR es significativamente mayor en pacientes con diagnóstico de PC y esta disminuye posterapia periodontal no quirúrgica. Además, se describe una asociación significativa entre la gravedad de la PC y la actividad de AR. Sin embargo, no se ha logrado aún establecer una relación causal clara, debido a otras variables causales comunes, por lo que se sugiere realizar estudios en relación a los mecanismos biológicos implicados y epidemiológicos en diferentes poblaciones.

\section{Referencias}

1. Alamanos Y, Voulgari PV, Drosos AA. Incidence and Prevalence of Rheumatoid Arthritis, Based on the 1987 American College of Rheumatology Criteria: A Systematic Review. Semin Arthritis Rheum 2006; 36 (3): 182-8.

2. Silman AJ, Pearson JE. Epidemiology and genetics of rheumatoid arthritis. Arthritis Res 2002; 4 (3): 265-72.

3. Alarcón AM, Muñoz S, Kaufman JS, Martínez C, Riedemann P, Kaliski S. Contribution of ethnic group and socieconomic status to degree of disability in rheumatoid arthitis. Rheumatol Int 2015; 35(4): 685-9.

4. Singh J, Saag K, Bridges L, Akl E, Bannuru R, Sullivan M et al. American College of Rheumatology Guideline for the Treatment of Rheumatoid Arthritis. Arthritis Care \& Research 2016; 68 (1): 1-25.

5. Bei $\mathrm{Xu}$, Jin Lin. Characteristics and risk factors of rheumatoid arthritis in the United States: an NHANES analysis. Peer J 2017; 24 (5): e4035.

6. Block SR. Hidden hazards and practical problems: comment on the 2002 update of the American College of Rheumatology Guidelines for the Management of Rheumatoid Arthritis. Arthritis Rheum 2002; 46 (11): 3102-3.

7. Van Riel PL. The development of the disease activity score (DAS) and the disease activity score using 28 joint counts (DAS28). Clin Exp Rheumatol 2014; 32 (85): 65-74.

8. Fernandes V, de Assis TM, Queiroz CC, de Figueiredo $\mathrm{PP}$, de Oliveira RU, da Silva NA. Use of biological therapies in rheumatoid arthritis management: a comparison between the main worldwide and brazilian recommendations. Rev Bras Reumatol 2011; 51 (3): 220-30.

9. Smolen JS, Aletaha D. Scores for all seasons: SDAI and CDAI. Clin Exp Rheumatol 2014; 32 (85): 75-9.

10. Hajishengallis G, Lamont RJ. Beyond the red complex and into more complexity: the polymicrobial synergy and dysbiosis (PSD) model of periodontal disease etiology. Mol Oral Microbiol 2012; 27 (6): 409-19.

11. Potempa J, Mydel P, Joanna Koziel J. The case for pe- riodontitis in the pathogenesis of rheumatoid arthritis. Nat Rev Rheumatol 2017; 13 (10): 606-20.

12. Gamonal J, Mendoza C, Espinoza I, Muñoz A, Urzúa I, Aranda W, et al. Clinical attachment loss in Chilean adult population: First Chilean National Dental Examination Survey. J Periodontol 2010; 81 (10): 1403-10.

13. Hermann KG, Backhaus M, Schneider U, Labs K, Loreck $\mathrm{D}$, Zühlsdorf S, et al. Rheumatoid arthritis of the shoulder joint: comparison of conventional radiography, ultrasound, and dynamic contrast-enhanced magnetic resonance imaging. Arthritis Rheum 2003; 48 (12): 3338-49.

14. McGraw WT, Potempa J, Farley D, Travis J. Purification, characterization, and sequence analysis of a potential virulence factor from Porphyromonas gingivalis, peptidylarginine deiminase. Infect Immun 1999; 67 (7): 3248-56.

15. Kimura Y, Yoshida S, Takeuchi T, Kimura M, Yoshikawa A, Hiramatsu Y, et al. Periodontal pathogens participate in synovitis in patients with rheumatoid arthritis in Clinical remission: a retrospective case-control study. Rheumatology (Oxford) 2015; 54 (12): 2257-63.

16. Konig MF, Abusleme L, Reinholdt J, Palmer RJ, Teles RP, Sampson K, et al. Aggregatibacter actinomycetemcomitans-induced hypercitrullination links periodontal infection to autoimmunity in rheumatoid arthritis. Sci Transl Med 2016; 8 (369): 369ra176.

17. Rosamma J, Sreeraj R, Sameera G, Binoy J. Association between chronic periodontitis and rheumatoid arthritis: a hospital-based case-control study. Rheumatol Int 2013; 33 (1): 103-9.

18. De Smit M, Westra J, Vissink A, Doornbos-van der Meer B, Brouwer E, Van Winkelhoff AJ. Periodontitis in established rheumatoid arthritis patients: a cross sectional clinical, microbiological and serological study. Arthritis Res Ther 2012; 14 (5): R222. doi: 10.1186/ ar4061.

19. Mikuls T, Payne J, Fang Yu, Thiele G, Reynolds R, Cannon G, et al. Periodontitis and Porphyromonas gingivalis in Patients with Rheumatoid Arthritis. Arthritis Rheumatol. 2014; 66(5): 1090-1100.

20. Lee JY, Choi IA, Kim JH, Kim KH, Lee EY, Lee EB, et al. Association between anti-Porphyromonas gingivalis or anti- $\alpha$-enolase antibody and severity of periodontitis or rheumatoid arthritis (RA) disease activity in RA. BMC Musculoskelet Disord 2015; 12 (16): 190.

21. Choi IA, Kim JH, Kim YM, Lee JY, Kim KH, Lee EY, et al. Periodontitis is associated with rheumatoid arthritis: a study with longstanding rheumatoid arthritis patients in Korea. Korean J Intern Med 2016; 31 (5): 977-86.

22. Mobini M, Maboudi A, Mohammadpour R. Periodon- 
titis in rheumatoid arthritis patients, abundance and association with disease activity. Med J Islam Repub Iran 2017; 10 (31): 44.

23. Chou YY, Lai KL, Chen DY, Lin CH, Chen HH. Rheumatoid Arthritis Risk Associated with Periodontitis Exposure: A Nationwide, Population-Based Cohort Study. PLoS One 2015; 10 (10): e0139693.

24. Hashimoto M, Yamazaki T, Hamaguchi M, Morimoto T, Yamori M, Asai K, et al. Periodontitis and Porphyromonas gingivalis in Preclinical Stage of Arthritis Patients. PLoS One 2015; 10 (4): e0122121.

25. Biyıkoğlu B, Buduneli N, Aksu K, Nalbantsoy A, Lappin DF, Evrenosoğlu E, et al. Periodontal therapy in chronic periodontitis lowers gingival crevicular fluid interleukin-1beta and DAS28 in rheumatoid arthritis patients. Rheumatol Int 2013; 33 (10): 2607-16.

26. Roman-Torres C, Neto J, Souza M, Schwartz-Filho H, Brandt W, Diniz R. An Evaluation of Non-Surgical Periodontal Therapy in Patients with Rheumatoid Arthritis. The Open Dentistry Journal 2015; 15 (9): 150-53.

27. Monsarrat P, Vergnes JN, Cantagrel A, Algans N, Cousty $S$, Kémoun P, et al. Effect of periodontal treatment on the Clinical parameters of patients with rheumatoid arthritis: study protocol of the randomized, controlled ESPERA trial. Trials 2013; 14 (1): 253.

28. Erciyas K, Sezer U, Ustün K, Pehlivan Y, Kisacik B, Senyurt SZ, et al. Effects of periodontal therapy on disease activity and systemic inflammation in rheumatoid arthritis patients. Oral Dis 2013; 19 (4): 394-400.

29. Shimada A, Kobayashi T, Ito S, Okada M, Murasawa A, Nakazono K, et al. Expression of anti-Porphyromonas gingivalis peptidylarginine deiminase immunoglobulin $\mathrm{G}$ and peptidylarginine deiminase- 4 in patients with rheumatoid arthritis and periodontitis. J Periodont Res 2016; 51 (1): 103-11.

30. Pinho M, Ribeiro R, Novaes A, Voltar JC. Relationship Between Periodontitis and Rheumatoid Arthritis and the Effect of Non-Surgical Periodontal Treatment. Braz Dent J 2009; 20 (5): 355-64.

31. Seror R, Le Gall-David S, Bonnaure-Mallet M, Schaeverbeke T, Cantagrel A, Minet J, et al. Association of Anti-Porphyromonas gingivalis Antibody Titers With Nonsmoking Status in Early Rheumatoid Arthritis. Arthritis \& Rheumatology 2015; (67) 7: 1729-37.

32. Quirke AM, Lugli E, Wegner N, Hamilton B, Charles P, Chowdhury M, et al. Heightened immune response to autocitrullinated Porphyromonas gingivalis peptidylarginine deiminase: a potential mechanism for breaching immunologic tolerance in rheumatoid arthritis. Ann Rheum Dis 2014; 73 (1): 263-69.

33. Kaur S, White S, Bartold P.M. Periodontal Disease and Rheumatoid Arthritis: A Systematic Review. J Dent Res. 2013; 92 (5): 399-408.

34. Kaur S, Bright R, Proudman SM, Bartold PM. Does periodontal treatment influence clinical and biochemical measures for rheumatoid arthritis? A systematic review and meta-analysis. Semin Arthritis Rheum 2014; 44 (2): 113-22.

35. Silvestre FJ, Silvestre J, Bagan J, Bagan JV. Effect of nonsurgical periodontal treatment in patients with periodontitis and rheumatoid arthritis: A systematic review. Med Oral Patol Oral Cir Bucal 2016; 21 (3): 349-54. 